\title{
Intraarterial delivery of virotherapy for glioblastoma
}

\author{
Visish M. Srinivasan, MD, ${ }^{1}$ Frederick F. Lang, MD, ${ }^{2}$ and Peter Kan, MD ${ }^{3}$ \\ 1Department of Neurosurgery, Barrow Neurological Institute, Phoenix, Arizona; ${ }^{2}$ Department of Neurosurgery, The University of \\ Texas MD Anderson Cancer Center, Houston, Texas; and ' ${ }^{2}$ epartment of Neurosurgery, University of Texas Medical Branch, \\ Galveston, Texas
}

\begin{abstract}
Oncolytic viruses (OVs) have been used in the treatment of cancer, in a focused manner, since the 1990s. These OVs have become popular in the treatment of several cancers but are only now gaining interest in the treatment of glioblastoma (GBM) in recent clinical trials. In this review, the authors discuss the unique applications of intraarterial (IA) delivery of OVs, starting with concepts of OV, how they apply to IA delivery, and concluding with discussion of the current ongoing trials. Several OVs have been used in the treatment of GBM, including specifically several modified adenoviruses. IA delivery of OVs has been performed in the hepatic circulation and is now being studied in the cerebral circulation to help enhance delivery and specificity. There are some interesting synergies with immunotherapy and IA delivery of OVs. Some of the shortcomings are discussed, specifically the systemic response to OVs and feasibility of treatment. Future studies can be performed in the preclinical setting to identify the ideal candidates for translation into clinical trials, as well as the nuances of this novel delivery method.
\end{abstract}

https://thejns.org/doi/abs/10.3171/2020.11.FOCUS20845

KEYWORDS endovascular; intraarterial; glioblastoma; virotherapy; oncolytic; stem cell

$\mathrm{O}$ NCOLYTIC viruses $(\mathrm{OVs})$ have been tested since the $1950 \mathrm{~s}^{1-3}$ however, it was not until the $1990 \mathrm{~s}^{1}$ that viral genomic engineering resulted in the first generation of cancer-selective therapeutic OVs. ${ }^{4}$ These selective therapeutic OVs are designed to selectively replicate within tumor cells, sparing normal tissue, and eventually cause oncolysis. Further research has demonstrated a complex downstream effect from viral therapy. Beyond direct oncolytic effects, there has been more of a focus recently on the involvement of the innate and adaptive immunity. Recognizing the strong tumor-mediated immunosuppression of glioblastoma (GBM), ${ }^{5,6}$ OVs have secondary effects of developing adaptive immunity that can result in lasting antitumor effects. ${ }^{4}$

Despite the promising nature of OVs, as discussed in this issue of Neurosurgical Focus, there are challenges in optimal delivery. Direct intratumoral delivery of viruses has been suboptimal due to backflow and limited viral distribution. However, in animal models, where delivery has been easier, successful cures have been achieved. Clearly, alternative delivery methods that distribute all of the viral dose widely throughout the tumor would be beneficial.
Intraarterial (IA) delivery of OVs has been established in other cancers and has had early applications in GBM as well. In this review, we will discuss the unique applications of IA delivery of OVs, starting with concepts of OV, how they apply to IA delivery, and concluding with discussion of the current ongoing trials.

\section{Oncolytic Virotherapy for Glioblastoma: How Far Have We Come?}

There are several OVs that have been studied for their potential in the treatment of GBM. This has included several types of OVs (for example, adenovirus, ${ }^{7}$ Newcastle disease virus, ${ }^{7}$ vaccinia virus, ${ }^{7}$ and herpes simplex virus $\left.[\mathrm{HSV}]^{8}\right)$, combinations thereof, and modified viruses such as the replication-deficient adenoviruses (e.g., $\mathrm{AdV}^{-} \mathrm{tk}^{4}$ ).

Adenovirus is among the best studied viruses for virotherapy. Its viral biology is well understood (independent of therapeutic value in GBM), and with gene modifications, it can be made into a conditionally replicative adenovirus. ${ }^{4}$ This has led to several "generations" of conditionally replicative adenoviruses, ${ }^{9}$ including Onyx- $015,{ }^{10}$ Delta-

ABBREVIATIONS BBB = blood-brain barrier; BTB = blood-tumor barrier; $\mathrm{CED}=$ convection-enhanced delivery; $\mathrm{CPI}=$ checkpoint inhibitor; $\mathrm{ESIA}=$ endovascular selective intraarterial; GBM = glioblastoma; HSV = herpes simplex virus; $I A=$ intraarterial; ICA = internal carotid artery; IL = interleukin; IV = intravenous; $M S C=$ mesenchymal stem cell; oHSV = oncolytic HSV; OV = oncolytic virus; PAMP = pathogen-associated molecular pattern.

SUBMITTED October 1, 2020. ACCEPTED November 16, 2020.

INCLUDE WHEN CITING DOI: 10.3171/2020.11.FOCUS20845. 
24-RGD, ${ }^{11}$ ICOVIR, ${ }^{12}$ and Delta-24-RGDOX.${ }^{13}$ These have been applied to cancers other than GBM as well, because the oncolytic function of these OVs and the tumor selectivity are not unique for GBM.

The agent that has advanced the furthest in clinical trials thus far has been Delta-24-RGD, which was the first clinical study to show direct oncolysis and replication of a therapeutic adenovirus in human brain tumors and to provide correlative evidence for a viral-induced antiglioma immune response. ${ }^{14}$ Since then, further trials have continued and have been partially reported on with Delta24-RGD. ${ }^{15}$

There have been a handful of cases of impressive results from oncolytic virotherapy, although none have been reported via IA methods yet. Gesundheit et al. recently shared 4 cases of patients treated with a combination of Newcastle disease virus, parvovirus, and vaccinia virus (all wild-type). ${ }^{16}$ These patients, 2 of whom had IDH wildtype GBM, all had no evidence of disease at follow-up, with 1 at 10 years of follow-up from initial OV therapy.

Closely related to OV, another area of virotherapy for gliomas has been gene therapy. The most common agent for this type of therapy has again been adenovirus, loaded with a variety of gene transfers to confer tumor resistance. Transfer of $p 53$, for example, via Ad-53 $3^{17}$ has been performed for recurrent gliomas in a phase 1 trial, demonstrating transduction of the gene into the nuclei of astrocytic tumor cells in patients and inducing apoptosis. However, the distribution of transfected cells has been limited to the peri-injection site, which on average is $5 \mathrm{~mm}$. While recent studies of gene therapy for GBM have been lacking, new delivery methods such as endovascular selective intraarterial (ESIA) may be considered.

\section{Rationale for IA Delivery}

The rationale for IA regional delivery of OVs has been established outside of the CNS. Experimental models have shown high efficacy for cancer, ${ }^{6}$ including for oral cancer $^{18}$ and liver cancer. ${ }^{19}$ This concept has been chosen to minimize systemic toxicities and exposure to neutralizing antibodies, the latter of which is a major concern for OVs.

OVs have had promising results for gliomas in the preclinical realm and to some extent in the clinical setting via stereotactic direct intratumoral injection. However, the results have been limited due to the viral distribution throughout the tumor. Lang et al. found that while there was successful DNX-2401 infection, replication, and killing of tumor cells, it followed the concentric zone pattern described in previous animal models..$^{14}$ This likely led to several patients in that trial without evidence of infection. This model shows that to reach maximal benefit and spread throughout the tumor, the injection cannula needs to be centered in the lesion, allowing consecutive replication. However, this limits the amount of virus that eventually reaches the edges of the tumor, which is precisely the area of tumor that is most challenging to resect and achieve tumor control. Studies of convection-enhanced delivery (CED) have tried to overcome this challenge, ${ }^{16}$ but it has possibly led to the failure of some phase 3 clinical trials employing CED.
Intravascular delivery of OVs has been performed. However, it is limited by the blood-brain barrier (BBB) and blood-tumor barrier (BTB), significantly limiting delivery and efficacy. Overcoming this limitation has been attempted by BBB disruption using mannitol to enhance IA delivery. One of the early uses of this for OV was described by Liu et al. in intracarotid delivery of an HSV vector (G47-delta) for breast cancer metastases to the brain..$^{20}$ G47-delta spread in the mouse breast cancer model was assessed by X-gal staining, demonstrating good spread throughout the tumor with the BBB disruption. However, the virus was also noted to distribute to the liver and lung (other studies estimated 2.6\%/g to the liver). Overall, these findings support the theory of IA delivery to enhance OV spread throughout the tumor, if the potential for systemic toxicity can be limited.

\section{IA Virotherapy and Gene Therapy for Other Cancers Hepatic Metastases}

IA infusion of viruses has been clinically investigated for cancers other than GBM. Hepatic arterial infusion of a selectively replicating OV, d11520 (Onyx-015), was performed in 2002 in a phase 2 trial by Reid et al. ${ }^{21}$ At that time, the enthusiasm around adenoviral therapies was limited by the contemporaneous report of a death from arterial infusion of an adenoviral gene therapy for ornithine transcarbamylase deficiency. For this application, they suggested the benefit of IA therapy for the diffuse/ multifocal nature of recurrent hepatocellular carcinoma after surgery and radiation therapy. These same theoretical benefits apply to GBM as well. They used a combination therapy of hepatic IA infusion of live virus combined with 5-FU and leucovorin for gastrointestinal metastases to the liver. Their main finding was the ability to use a lower dose of live virus for safety (one-tenth of the dose used in the gene therapy trial), which was well tolerated. This induced a cyclical/recurrent viremia consistent with viral replication, proinflammatory cytokine induction, and evidence for chemosensitization of refractory tumors. In addition, this study was the first to demonstrate clinical replication of a therapeutic virus delivered by an IA route, with a subsequent rapid clearing of the virus from the blood. They concluded their paper with a goal of future studies to focus on "improving the efficiency of vascular delivery and on improving the replication and potency of the therapeutic virus."

\section{Head and Neck Cancer}

Another area of clinical evaluation of OVs has been in head and neck cancer. In 2009, Xu et al. ${ }^{59}$ reported on a phase 1 clinical trial with AdV-tk/ganciclovir therapy in 18 patients with nasopharyngeal carcinoma. They demonstrated that AdV-tk gene therapy could be administered safely, although there were systemic symptoms (fever mostly) with systemic administration. They later wanted to translate this to treatment of high-grade gliomas. After demonstrating the intratumoral injection in the nasopharyngeal carcinoma, they followed this with an IA strategy to achieve dose intensification and localized, concentrated delivery. In 53 patients who underwent IA cerebral infu- 
sion of AdV-tk, they achieved improved median progression-free survival (29.6 vs 8.4 weeks).

Additional gene therapy trials have been performed for head and neck cancer more recently as well. Li et al. reported in $2014^{60}$ on 99 patients enrolled in a phase 3 randomized, placebo-controlled, double-blind trial, in which they found a better response with IA therapy (recombinant adenovirus-p53 [rAD-p53] gene therapy) with chemotherapy combined, rather than with each of the treatments alone with a placebo of the other. Furthermore, they confirmed the mechanistic success of this treatment: rAd-p53 treatment increased Bax expression in the primary tumor in $80 \%$ of the patients.

\section{Endovascular Selective Intraarterial Therapy}

ESIA therapy or infusion has been used to deliver therapeutic agents to brain tumors. ${ }^{18,20}$ This concept has been most successfully applied in retinoblastoma, ${ }^{22}$ but has also been evaluated in GBM. ESIA therapy of retinoblastoma is increasingly accepted as a treatment option over enucleation. It developed from the initial open surgical carotid infusion of triethylenemelamine nitrogen mustard, to endovascular infusion of melphalan (L-phenylalanine mustard $)^{23}$ into the internal carotid artery (ICA) with distal balloon occlusion, to now direct catheterization and infusion $^{24}$ of melphalan, carboplatin, and topotecan through the ophthalmic artery, with a reported $92 \%$ ocular survival in 452 eyes. ${ }^{25}$

Thus far, the therapeutic agent has been chemotherapy, including older agents such as cisplatin/vincristine, and more recently "newer" agents such as temozolomide ${ }^{26}$ and bevacizumab. ${ }^{27,28}$ These agents have had some limited success, but have not progressed beyond phase 2 studies (www.clinicaltrials.gov no.: NCT01811498). IA therapy has also been performed for another common oncological therapy outside of the CNS (brachytherapy). ${ }^{29}$ Pasciak et al. ${ }^{29}$ evaluated yttrium-90 $\left({ }^{90} \mathrm{Y}\right)$ in a canine model of GBM. A microcatheter technique was used to selectively catheterize the ICA, middle cerebral artery, or posterior cerebral artery in the animals, followed by IA infusion of ${ }^{90} \mathrm{Y}$ glass microspheres. Radiation was absorbed into healthy and tumoral tissue, and at 1 month posttherapy, "patient" animals showed a 24\%-94\% reduction in mass volume; further studies are pending.

The next generation of therapies for GBMs, which includes OVs, has the potential to overcome some of the mechanistic limitations of these chemotherapies as well as their dose-related side effects. ESIA therapy can theoretically be used with a variety of classes of agents, ${ }^{28}$ including $\mathrm{OVs}^{30}$ (Fig. 1). One of the ways to overcome the $\mathrm{BBB} / \mathrm{BTB}$ in delivery is to use mesenchymal stem cells (MSCs).

\section{The Role of MSCs in IA Delivery}

Based on findings of Nakamizo et al. that MSCs have tropism to human gliomas, ${ }^{31}$ Yong et al. then studied MSCs as a delivery vehicle for Delta-24-RGD. ${ }^{32}$ MSCs were transfected with Delta-24 and then delivered via intracarotid injection in a mouse xenograft model of GBM. In this study, they found that MSCs were required for Del-
ta-24-RGD delivery to the gliomas; intracarotid delivery of free Delta-24 did not deposit into the virus, consistent with the non-BBB disruption arm of the Liu et al. study. ${ }^{20}$ The MSC-Delta-24 construct led to localization within gliomas, spread of Delta-24 into the glioma cells evenly throughout the tumor, and inhibition of xenograft growth with resultant improvement in mouse survival.

This preclinical work, as well as further studies that describe the mechanisms underlying MSC tropism for gliomas, established the concept of IA delivery. ${ }^{33}$ Intravenous (IV) delivery is certainly easier from a clinical standpoint. However, studies of IV MSC delivery have been hampered by pulmonary passage, resulting in a marked reduction of the eventual MSC delivery at the target. ${ }^{34}$ Additionally, there have been concerns about the increased potential for neutralizing antibodies via IV administration, which can limit the effective dose of the virus and secondary immune response. Thus, MSCs provide "shielding" from the potential humoral antiviral response until the virus can be delivered to the tumor in high doses, in addition to its assistance in crossing the BBB (Table 1).

IA delivery of MSCs loaded with OVs (Delta-24) has been studied in an in vitro endovascular mode ${ }^{35}$ as well as in a large animal canine model. ${ }^{36}$ These experiments have shown that IA microcatheter delivery of MSCs carrying OV retains the oncolytic effects of the virus as well as the tropism of the MSCs. Such delivery is quite robust, unaffected by various catheter factors (positioning/shape, infusion speed, or catheter type). When this method was performed in a canine model, it was found to be safe in the anterior circulation without dose-related toxicity. This is currently being evaluated in a phase 1 clinical trial of ESIA therapy with Delta-24 MSCs (www.clinicaltrials. gov no.: NCT03896568).

Other types of stem cells, including neural stem cells, have been considered and evaluated in animal models. ${ }^{37}$ However, MSCs are easier to harvest, can be used as either autologous or allogeneic types, have fewer ethical issues surrounding their use, and can easily be cultured in vitro. ${ }^{37,38}$

Oncolytic HSV (oHSV) is another agent with potential for ESIA-MSC-OV therapy, as has been suggested by Shah. ${ }^{30}$ Whereas some clinical studies of oHSV used in the resection bed of GBMs resulted in "washout," ESIA infusion with loading in MSCs and proapoptotic variant oHSV-TRAIL (tumor necrosis factor-related apoptosisinducing ligand) may overcome these shortcomings. ${ }^{28}$

Other methods of using OVs can also be considered. Similar to conventional IA delivery, ESIA delivery can be performed with replication-selective viruses with therapeutic transgenes. ESIA delivery offers the advantage of increased specificity of delivery, especially if combined with MSCs. Although preclinical data are lacking thus far, these are areas of potential investigation. Examples of such therapeutic payload-carrying viruses that have been used for GBM include TOCA 511 and TG6002, which carry cytosine deaminase- or 5-fluoro-uracil-producing genes, respectively. These have thus far been tested via IV infusion with follow-up oral administration. 


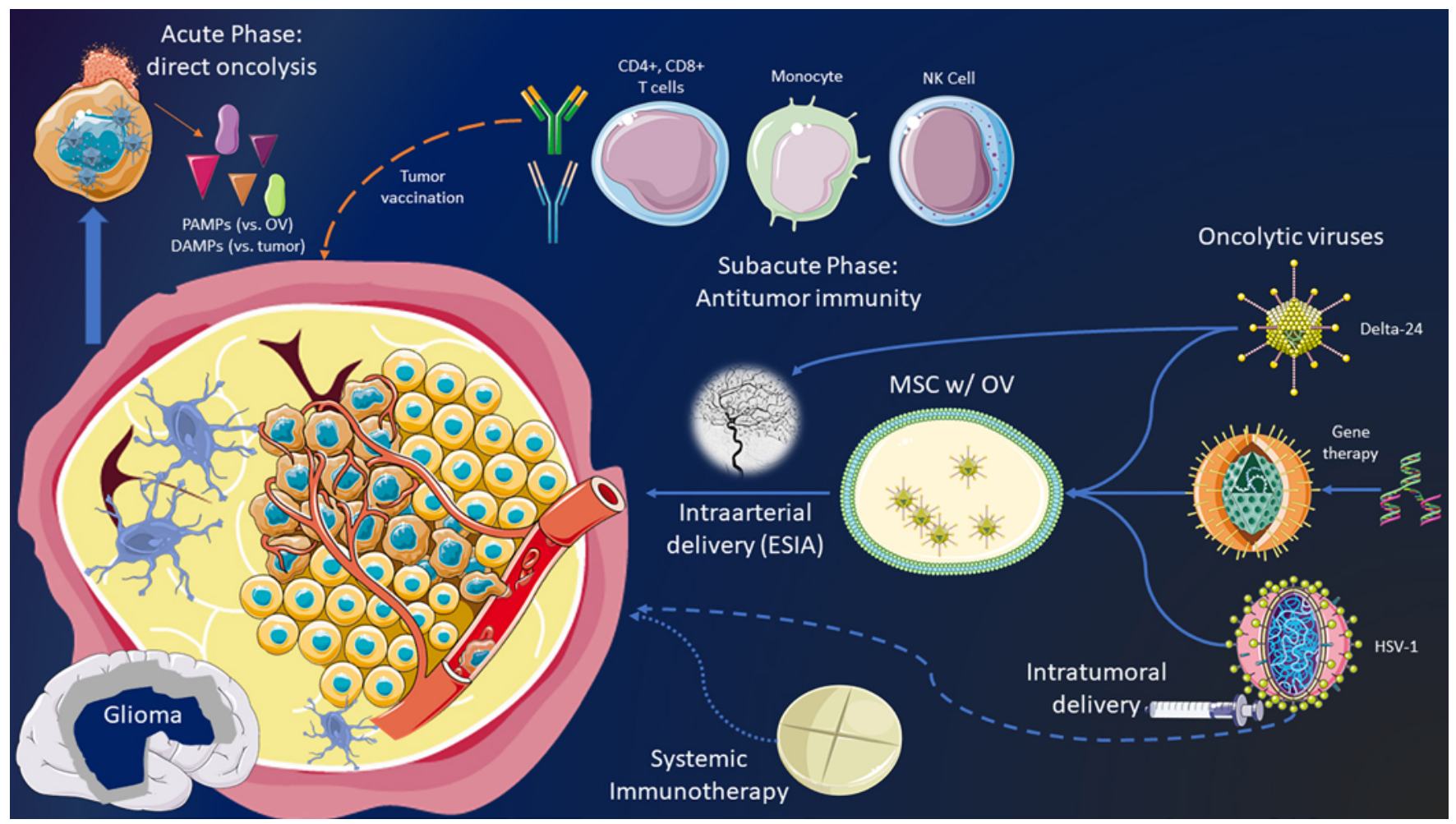

FIG. 1. Mechanisms of IA and other OV therapy. Various OVs have been developed for tumor selectivity (such as Delta-24-RGD). These can also carry therapeutic transgenes (i.e., gene therapy). These viruses can be delivered intraarterially (solid blue line), either as naked virus or in an MSC carrier ("MSC w/ OV") that provides additional tumor selectivity through tropic mechanisms. This can be performed via intracarotid delivery or using the ESIA technique. Direct intratumoral delivery (long dashed blue line) can also be performed, usually via a stereotactically placed needle or using CED. Systemic immunotherapy (e.g., a CPI) or other chemotherapies can be used in combination with OV (short dotted blue line). A glioma with its enhanced vasculature is noted, which can be used for ESIA delivery. Direct oncolysis is caused by viral replication within tumor cells. This results in the generation of neoantigens, from both the virus itself and tumor cells: PAMPs and damage-associated molecular patterns (DAMPs). These stimulate inflammatory responses, with tumor-directed antibodies and an increased immune infiltrate consisting of NK cells, activated T cells, and monocytes, producing a longer, sustained subacute antitumor immune response. Created using Servier Medical Art by Servier, licensed under Creative Commons Attribution 3.0 (https://creativecommons.org/licenses/by/3.0/). Copyright Visish M. Srinivasan. Published with permission.

\section{Challenges in Immunotherapy}

Both the promise of and the challenges for immunotherapy for GBM are related to the immunosuppressive environment, as GBM enables its proliferation by alteration of its microenvironment to support immune evasion. Tumor immunosuppression is mediated by transforming growth factor- $\beta$, vascular endothelial growth factor, prostaglandin $\mathrm{E}_{2}$, and interleukin (IL)-10, which results in T-cell depletion and suppression of T-cell cytotoxicity. ${ }^{39}$ Thus, the potential of activating the immune system by checkpoint inhibitors (CPIs) and other immunotherapies is promising..$^{40}$ However, monotherapy with such therapies in the CNS, with its immune privilege, is less optimal as there is no activation stimulus. Thus, in the aftermath of the limited results of CPI monotherapy, there has been interest in combination therapy (discussed below). Challenges in designing and executing trials for a variety of immunotherapies have been reviewed extensively. ${ }^{41}$ These other challenges include modulation of the immune response, management of cerebral edema and dexamethasone, and minimizing immune-related adverse effects. Additional challenges include dynamic genetic mutation ${ }^{42}$ heterogeneity ${ }^{43}$ and changes over the course of treatment. ${ }^{44,45}$ Thus, treatments such as OV that are able to generate a lasting proimmune effect or have mechanisms independent of specific mutations are desirable.

\section{Benefits of OV}

There are several benefits to OV usage in GBM, some of which are particularly salient for IA or ESIA infusion compared to other agents. The first is selective replication; the selective nature of OVs allows a reduction in potential side effects as compared to IA delivery of chemotherapies, which may have diminished side effects intraarterially versus intravenously, but are still nonetheless active in the systemic circulation. ESIA delivery of OVs is theoretically ready for translation, given that other delivery mechanisms have shown some clinical value via intratumoral injection $^{14}$ or CED. The establishment of clinical safety here is key, although ESIA OV trials still need to undergo phase 
TABLE 1. Benefits and drawbacks of IA therapy of OVs for GBM

\begin{tabular}{ll}
\hline \multicolumn{1}{c}{ Benefits } & \multicolumn{1}{c}{ Drawbacks } \\
\hline Minimally invasive delivery & $\begin{array}{c}\text { Requires neurointervention expertise and inpatient setting; increased cost of } \\
\text { delivery compared to IV delivery }\end{array}$ \\
\hline Potential for repeat delivery & Repeat infusion still more involved compared to IV chemotherapy \\
\hline Focused delivery of virus to tumor bed & \\
\hline Decreased systemic response & May require "shielding" of virus from humoral antiviral factors \\
\hline Can be used in conjunction w/ MSCs to enhance delivery & \\
\hline Opening of BBB to increase effective dose &
\end{tabular}

1 testing to determine the maximum tolerated dose and unique safety of each agent. OVs generally target common and early mutations that affect nearly all tumor cells, such as $p 53$ or retinoblastoma $(R b)$ mutations. This gives them efficacy against a host of tumors other than GBM, but also enables them to overcome the mutational heterogeneity that characterizes GBM. ${ }^{46}$

Tumor control via OV has been described in an early and late response. ${ }^{47}$ The use of MSCs in these cases as a carrier can also alter the response and immunological response in some ways. In the early response, there is direct tumor lysis by intracellular replication of OV. This can be enhanced by the addition of various moieties to the virus to enhance binding and affinity (e.g., the addition of RGD to the Delta-24 virus). The distribution of virus for this early phase can be theoretically enhanced by ESIA, also perhaps with the use of MSCs. The early phase is also characterized by tumor and MSC-mediated immunosuppression, which allows efficient viral replication. As this wears off, there is a late-phase response. ${ }^{13}$ This is characterized by antiviral leading to antitumoral immunity. ${ }^{47}$ The immune responses are elicited by pathogen-associated molecular patterns (PAMPS; e.g., viral capsid) and tumor milieu-associated molecular pattens. In this manner, the virus can essentially act as an active antitumor vaccine. GBM is known to have limited immunogenicity, with few known tumor-restricted antigens. ${ }^{41}$ This function of OVs in producing an immune response enables their effect as monotherapy as well as combination therapy (discussed later). This local inflammatory response is believed to then lead to adaptive antiviral and antitumoral immunity. These understood mechanisms of OV response need to be understood better in the context of a novel delivery method (IA/ESIA infusion), and then subsequently can be harnessed for the unique potential of this delivery (potentially, targeted/repeated infusions).

\section{Potential for Combination Therapy}

There is potential for combination therapies using ESIA infusion of OV, with other adjuncts such as traditional chemotherapy, CPIs, and/or radiation. Among these, there is some evidence to suggest that CPIs have some synergy with OVs. This can be extrapolated to include use with IA OV therapy.

CPIs for GBM, as studied recently in GBM with antiPD-1 nivolumab, ${ }^{40}$ showed early promise but have been limited by the immunosuppressive environment of GBM.
This might potentially be overcome by use of combination therapy. ${ }^{48}$ The concept is well described in a review by Jiang et al. (Fig. 2). ${ }^{49}$ The treatment choice between OV and CPI can be chosen based on the patient immune response and the immune checkpoint expression level. Combination therapy may be particularly helpful and/or required for patients with low-immunogenicity tumors (most GBMs), a weak cohort of Th1-primed lymphocytes, and high levels of expression of immune checkpoints in the tumor infiltrative T cells (Fig. 3). ${ }^{49}$ Many of the combination therapies discussed have theoretical advantages but still require preclinical validation.

CPIs have been used in combination therapy ${ }^{50}$ including with OVs. ${ }^{51}$ The goal here is to increase the immunogenicity of GBM. Currently, the only ongoing trial with OV-CPI is with DNX-2401 and pembrolizumab (www. clinicaltrials.gov no.: NCT02798406). Delta-24-RGD treatment for GL261 murine GBM was shown to change the recruitment of macrophages, NK cells, and CD4+ and CD8+ T cells to the tumor site..$^{52-54}$ Thus, modification of the immune microenvironment by adenoviral therapy of high-grade gliomas can enhance the efficacy of immunotherapy, providing some preclinical basis for clinical testing of this combination therapy.

The use of combination therapies has been suggested in GBM to overcome tumor cross-resistance. When combination therapies use agents that have radically different mechanisms with limited overlapping toxicities, they are additionally likely to be tolerated and avoid resistance. ${ }^{21,55,56}$

Finally, IA infusion allows for easy repeat treatment with therapeutic OVs compared to alternative methods of delivery that require surgery (such as direct infusion and CED). Given the relative novelty of ESIA, this has not yet been studied, even in the preclinical arena. However, there is precedent for repeated IA treatment in retinoblastoma ${ }^{57}$ and in liver metastases. ${ }^{21,58}$ When used in a phase 2 clinical trial for gastrointestinal liver metastases, repeat infusion was found to have delayed secondary peaks of viremia, overcoming the presence of neutralizing antibody titers and antiviral cytokines (e.g., IL-6). Specific to the Delta-24-RGD, repeated intratumoral delivery has been found to have additional therapeutic value. Thus, it may theoretically help to extend the early-phase direct oncolytic effects of OVs, to be followed later by immune-mediated tumor clearance. 


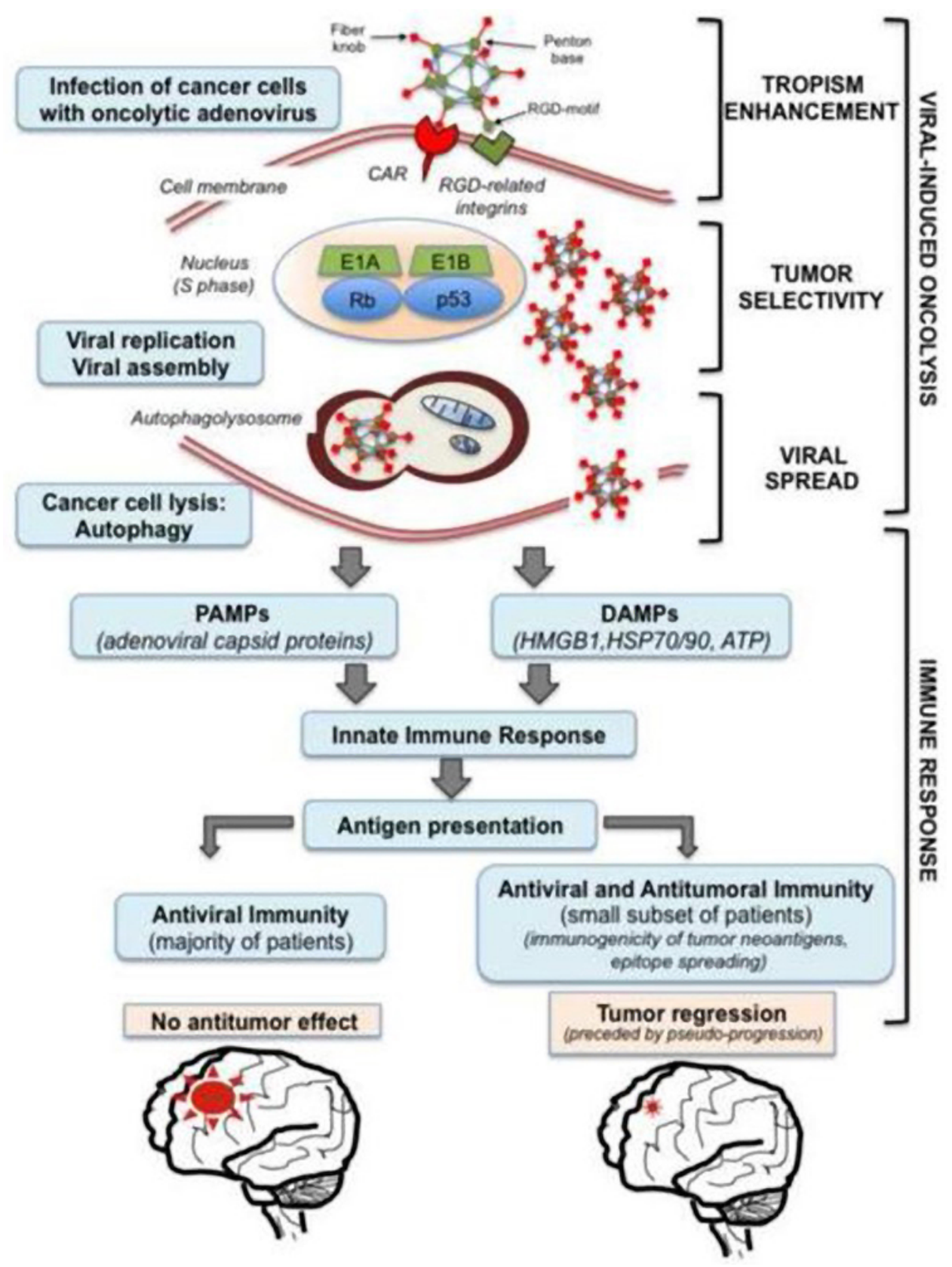

FIG. 2. Anticancer effect by OVs comes in two stages, i.e., viral replication followed by the immune response. Stage 1 is optimized with enhanced tumor tropism, cancer-cell specificity, and increased interaction with cell proteins to accelerate lysis. The immune response is composed of antitumor and antiviral immune responses, elicited by the accumulation of PAMPs (e.g., adenovirus capsid proteins), together with the release to the tumor milieu of DAMPs, which are responsible for the attraction of innate immune cells and eventually for T-cell priming. This sustained antitumor response can allow for tumor regression even after eventual viral clearance. It is possible that tumor regression occurs after adenoviral infection. Reprinted from Curr Opin Virol Vol 13, Jiang H, Gomez-Manzano C, Rivera-Molina Y, Lang FF, Conrad CA, Fueyo J: Oncolytic adenovirus research evolution: from cell-cycle checkpoints to immune checkpoints. pp 33-39, 2015, with permission from Elsevier.

\section{Conclusions}

Oncolytic virotherapy is a promising concept for treatment of malignant glioma and has already been used successfully in early clinical studies. IA therapy offers an alternative method of delivery of this selective virus into the tumor bed. Future studies can be performed in the pre- clinical setting to identify the ideal candidates for translation into clinical trials, as well as the nuances of this novel delivery method.

\section{Acknowledgments}

This study was supported by the National Cancer Institute 


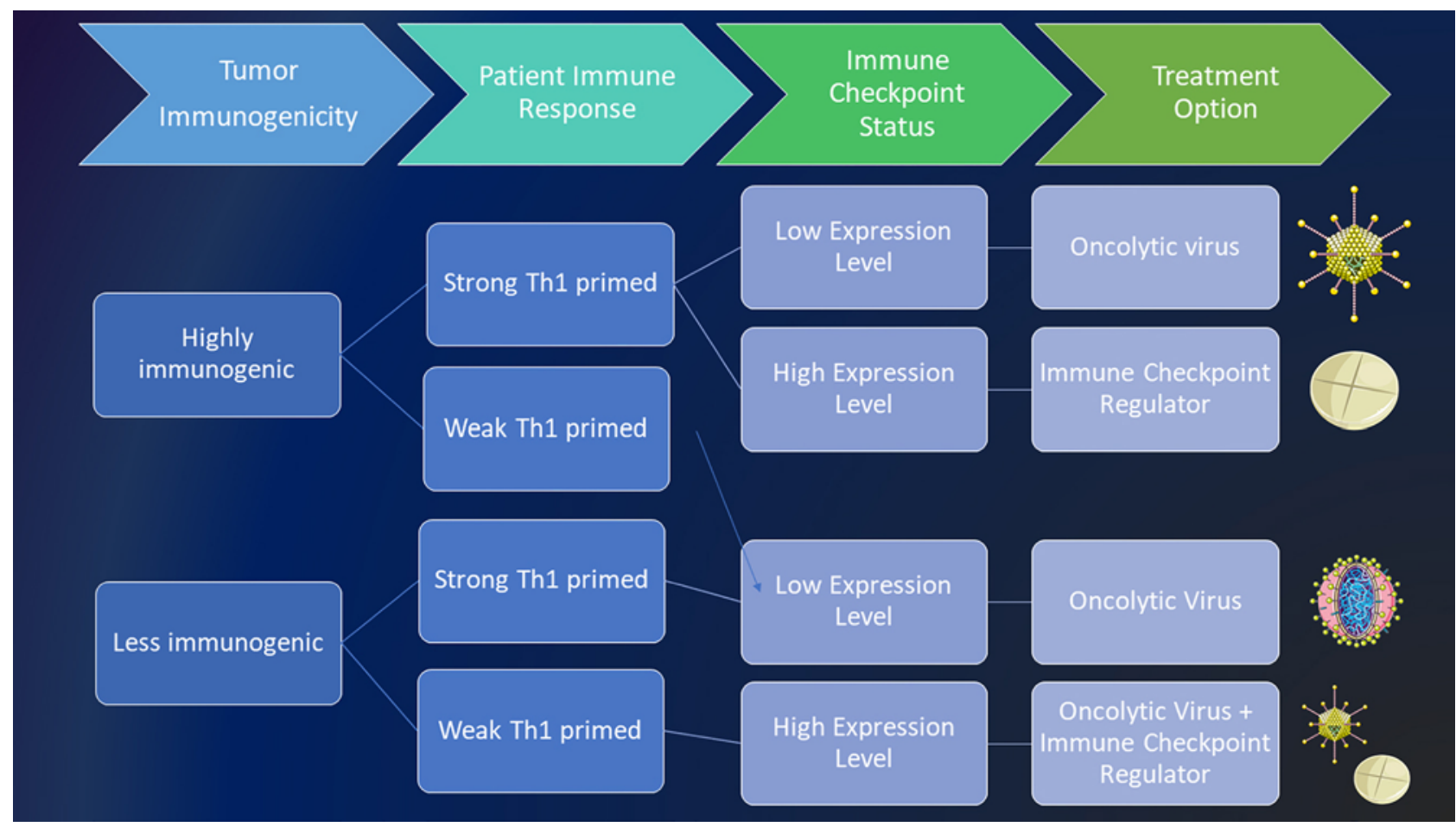

FIG. 3. Illustration showing the rationale for the combination of oncolytic adenoviruses with immune checkpoint modulators. This demonstrates the manner in which combination therapy can be tailored to a patient's particular tumor immunogenicity and the patient's relative response. Combination therapy may be best for patients with weak Th1-primed lymphocytes, high levels of expression of immune checkpoints in the tumor infiltrative T cells, and a low degree of immunogenicity. Modified from Curr Opin Virol Vol 13, Jiang H, Gomez-Manzano C, Rivera-Molina Y, Lang FF, Conrad CA, Fueyo J: Oncolytic adenovirus research evolution: from cell-cycle checkpoints to immune checkpoints. pp 33-39, 2015, with permission from Elsevier. Created using Servier Medical Art by Servier, licensed under Creative Commons Attribution 3.0 (https://creativecommons.org/licenses/by/3.0/).

(grant nos. 1R01CA214749, 1R01CA247970, and 2P50CA127001), The University of Texas MD Anderson Moon Shots Program, the Broach Foundation for Brain Cancer Research, the Elias Family Fund, the Priscila and Jason Hiley Fund, the Baumann Family/ Curefest Fund, the Jim and Pam Harris Fund, the Gene Pennebaker Brain Cancer Fund, the Schneider Memorial Fund, the Sweet Family Cancer Research Fund, the Dr. Marnie Rose Foundation, the Gold Family Memorial Fund, and the Sorenson Foundation (all to Dr. Lang).

\section{References}

1. Alemany R. Viruses in cancer treatment. Clin Transl Oncol. 2013;15(3):182-188.

2. Kelly E, Russell SJ. History of oncolytic viruses: genesis to genetic engineering. Mol Ther. 2007;15(4):651-659.

3. Larson C, Oronsky B, Scicinski J, et al. Going viral: a review of replication-selective oncolytic adenoviruses. Oncotarget. 2015;6(24):19976-19989.

4. Kiyokawa J, Wakimoto H. Preclinical and clinical development of oncolytic adenovirus for the treatment of malignant glioma. Oncolytic Virother. 2019;8:27-37.

5. Ferguson SD, Srinivasan VM, Heimberger AB. The role of STAT3 in tumor-mediated immune suppression. J Neurooncol. 2015;123(3):385-394.

6. Ma Q, Long W, Xing C, et al. Cancer stem cells and immunosuppressive microenvironment in glioma. Front Immunol. 2018;9:2924.

7. Alkassar M, Gärtner B, Roemer K, et al. The combined effects of oncolytic reovirus plus Newcastle disease virus and reovirus plus parvovirus on U87 and U373 cells in vitro and in vivo. J Neurooncol. 2011;104(3):715-727.

8. Chiocca EA, Nakashima H, Kasai K, et al. Preclinical toxicology of rQNestin34.5v.2: an oncolytic herpes virus with transcriptional regulation of the ICP34.5 neurovirulence gene. Mol Ther Methods Clin Dev. 2020;17:871-893.

9. Rius-Rocabert S, García-Romero N, García A, et al. Oncolytic virotherapy in glioma tumors. Int J Mol Sci. 2020;21(20):7604.

10. Bischoff JR, Kirn DH, Williams A, et al. An adenovirus mutant that replicates selectively in p53-deficient human tumor cells. Science. 1996;274(5286):373-376.

11. Fueyo J, Gomez-Manzano C, Alemany R, et al. A mutant oncolytic adenovirus targeting the $\mathrm{Rb}$ pathway produces antiglioma effect in vivo. Oncogene. 2000;19(1):2-12.

12. Alonso MM, Cascallo M, Gomez-Manzano C, et al. ICOVIR-5 shows E2F1 addiction and potent antiglioma effect in vivo. Cancer Res. 2007;67(17):8255-8263.

13. Jiang H, Rivera-Molina Y, Gomez-Manzano C, et al. Oncolytic adenovirus and tumor-targeting immune modulatory therapy improve autologous cancer vaccination. Cancer Res. 2017;77(14):3894-3907.

14. Lang FF, Conrad C, Gomez-Manzano C, et al. Phase I study of DNX-2401 (Delta-24-RGD) oncolytic adenovirus: replication and immunotherapeutic effects in recurrent malignant glioma. J Clin Oncol. 2018;36(14):1419-1427.

15. Zadeh G, Lang F, Daras M, et al. ATIM-24. Interim results of a phase II multicenter study of the conditionally replicative oncolytic adenovirus DNX-2401 with pembrolizumab (KEYTRUDA) for recurrent glioblastoma; captive study (Keynote-192). Neuro Oncol. 2018;20(suppl 6):vi6. 
16. Gesundheit B, Ben-David E, Posen Y, et al. Effective treatment of glioblastoma multiforme with oncolytic virotherapy: a case-series. Front Oncol. 2020;10:702.

17. Lang FF, Bruner JM, Fuller GN, et al. Phase I trial of adenovirus-mediated p53 gene therapy for recurrent glioma: biological and clinical results. J Clin Oncol. 2003;21(13): $2508-2518$.

18. Stiles BM, Bhargava A, Adusumilli PS, et al. The replicationcompetent oncolytic herpes simplex mutant virus NV1066 is effective in the treatment of esophageal cancer. Surgery. 2003;134(2):357-364.

19. Kooby DA, Carew JF, Halterman MW, et al. Oncolytic viral therapy for human colorectal cancer and liver metastases using a multi-mutated herpes simplex virus type-1 (G207). FASEB J. 1999;13(11):1325-1334.

20. Liu R, Martuza RL, Rabkin SD. Intracarotid delivery of oncolytic HSV vector G47 $\Delta$ to metastatic breast cancer in the brain. Gene Ther. 2005;12(8):647-654.

21. Reid T, Galanis E, Abbruzzese J, et al. Hepatic arterial infusion of a replication-selective oncolytic adenovirus (d11520): phase II viral, immunologic, and clinical endpoints. Cancer Res. 2002;62(21):6070.

22. Ravindran K, Dalvin LA, Pulido JS, Brinjikji W. Intra-arterial chemotherapy for retinoblastoma: an updated systematic review and meta-analysis. J Neurointerv Surg. 2019;11(12): $1266-1272$.

23. Reese AB, Hyman GA, Merriam GR Jr, et al. Treatment of retinoblastoma by radiation and triethylenemelamine. AMA Arch Opthalmol. 1954;53(4):505-513.

24. Yamane T, Kaneko A, Mohri M. The technique of ophthalmic arterial infusion therapy for patients with intraocular retinoblastoma. Int J Clin Oncol. 2004;9(2):69-73.

25. Francis JH, Levin AM, Zabor EC, et al. Ten-year experience with ophthalmic artery chemosurgery: ocular and recurrence-free survival. PLoS One. 2018;13(5):e0197081.

26. Shin BJ, Burkhardt J-K, Riina HA, Boockvar JA. Superselective intra-arterial cerebral infusion of novel agents after blood-brain disruption for the treatment of recurrent glioblastoma multiforme: a technical case series. Neurosurg Clin $N$ Am. 2012;23(2):323-329, ix-x.

27. Boockvar JA, Tsiouris AJ, Hofstetter CP, et al. Safety and maximum tolerated dose of superselective intraarterial cerebral infusion of bevacizumab after osmotic blood-brain barrier disruption for recurrent malignant glioma. J Neurosurg. 2011;114(3):624-632.

28. Srinivasan VM, Lang FF, Chen SR, et al. Advances in endovascular neuro-oncology: endovascular selective intraarterial (ESIA) infusion of targeted biologic therapy for brain tumors. J Neurointerv Surg. 2020;12(2):197-203.

29. Pasciak AS, Manupipatpong S, Hui FK, et al. Yttrium-90 radioembolization as a possible new treatment for brain cancer: proof of concept and safety analysis in a canine model. EJNMMI Res. 2020;10(1):96.

30. Shah K. Stem cell-based therapies for tumors in the brain: are we there yet? Neuro Oncol. 2016;18(8):1066-1078.

31. Nakamizo A, Marini F, Amano T, et al. Human bone marrow-derived mesenchymal stem cells in the treatment of gliomas. Cancer Res. 2005;65(8):3307-3318.

32. Yong RL, Shinojima N, Fueyo J, et al. Human bone marrowderived mesenchymal stem cells for intravascular delivery of oncolytic adenovirus Delta24-RGD to human gliomas. Cancer Res. 2009;69(23):8932-8940.

33. Shinojima N, Hossain A, Takezaki T, et al. TGF- $\beta$ mediates homing of bone marrow-derived human mesenchymal stem cells to glioma stem cells. Cancer Res. 2013;73(7):2333-2344

34. Fischer UM, Harting MT, Jimenez F, et al. Pulmonary passage is a major obstacle for intravenous stem cell delivery: the pulmonary first-pass effect. Stem Cells Dev. 2009;18(5): 683-692.
35. Srinivasan VM, Gumin J, Camstra KM, et al. Microcatheter delivery of neurotherapeutics: compatibility with mesenchymal stem cells. J Neurosurg. 2020;133(4):1182-1190.

36. Srinivasan VM, Gumin J, Camstra KM, et al. Endovascular selective intra-arterial infusion of mesenchymal stem cells loaded with Delta-24 in a canine model. Neurosurgery. 2020; 88(1):E102-E113.

37. Parker Kerrigan BC, Shimizu Y, Andreeff M, Lang FF. Mesenchymal stromal cells for the delivery of oncolytic viruses in gliomas. Cytotherapy. 2017;19(4):445-457.

38. Pittenger MF, Mackay AM, Beck SC, et al. Multilineage potential of adult human mesenchymal stem cells. Science. 1999;284(5411):143-147.

39. Roszman T, Elliott L, Brooks W. Modulation of T-cell function by gliomas. Immunol Today. 1991;12(10):370-374.

40. Reardon DA, Brandes AA, Omuro A, et al. Effect of nivolumab vs bevacizumab in patients with recurrent glioblastoma: the CheckMate 143 phase 3 randomized clinical trial. JAMA Oncol. 2020;6(7):1003-1010.

41. Weathers S-P, Gilbert MR. Current challenges in designing GBM trials for immunotherapy. J Neurooncol. 2015;123(3): 331-337.

42. Chen J, McKay RM, Parada LF. Malignant glioma: lessons from genomics, mouse models, and stem cells. Cell. 2012; 149(1):36-47.

43. Brennan CW, Verhaak RGW, McKenna A, et al. The somatic genomic landscape of glioblastoma. Cell. 2013;155(2):462-477.

44. Mansouri A, Karamchandani J, Das S. Molecular genetics of secondary glioblastoma. In: De Vleeschouwer S, ed. Glioblastoma. Codon Publications; 2017:27-42.

45. Khalafallah AM, Huq S, Jimenez AE, et al. "Zooming in" on glioblastoma: understanding tumor heterogeneity and its clinical implications in the era of single-cell ribonucleic acid sequencing. Neurosurgery. Published online July 16, 2020. doi:10.1093/neuros/nyaa305

46. Patel AP, Tirosh I, Trombetta JJ, et al. Single-cell RNA-seq highlights intratumoral heterogeneity in primary glioblastoma. Science. 2014;344(6190):1396-1401.

47. Gras Navarro A, Björklund AT, Chekenya M. Therapeutic potential and challenges of natural killer cells in treatment of solid tumors. Front Immunol. 2015;6:202.

48. Filley AC, Henriquez M, Dey M. Recurrent glioma clinical trial, CheckMate-143: the game is not over yet. Oncotarget. 2017;8(53):91779-91794.

49. Jiang H, Gomez-Manzano C, Rivera-Molina Y, et al. Oncolytic adenovirus research evolution: from cell-cycle checkpoints to immune checkpoints. Curr Opin Virol. 2015;13:33-39.

50. Kim JE, Patel MA, Mangraviti A, et al. Combination therapy with Anti-PD-1, Anti-TIM-3, and focal radiation results in regression of murine gliomas. Clin Cancer Res. 2017;23(1): 124-136.

51. McGranahan T, Therkelsen KE, Ahmad S, Nagpal S. Current state of immunotherapy for treatment of glioblastoma. Curr Treat Options Oncol. 2019;20(3):24.

52. Jiang H, Clise-Dwyer K, Ruisaard KE, et al. Delta-24-RGD oncolytic adenovirus elicits anti-glioma immunity in an immunocompetent mouse model. PLoS One. 2014;9(5):e97407.

53. Kleijn A, Kloezeman J, Treffers-Westerlaken E, et al. The in vivo therapeutic efficacy of the oncolytic adenovirus Delta24-RGD is mediated by tumor-specific immunity. PLoS One. 2014;9(5):e97495.

54. Kleijn A, Kloezeman J, Treffers-Westerlaken E, et al. The therapeutic efficacy of the oncolytic virus Delta24-RGD in a murine glioma model depends primarily on antitumor immunity. Oncoimmunology. 2014;3(9):e955697.

55. Kemeny N, Huang Y, Cohen AM, et al. Hepatic arterial infusion of chemotherapy after resection of hepatic metastases from colorectal cancer. N Engl J Med. 1999;341(27):20392048. 
56. Martuza RL, Malick A, Markert JM, et al. Experimental therapy of human glioma by means of a genetically engineered virus mutant. Science. 1991;252(5007):854-856.

57. Gobin YP, Dunkel IJ, Marr BP, et al. Intra-arterial chemotherapy for the management of retinoblastoma: four-year experience. Arch Ophthalmol. 2011;129(6):732-737.

58. Sze DY, Iagaru AH, Gambhir SS, et al. Response to intraarterial oncolytic virotherapy with the herpes virus NV1020 evaluated by $\left[{ }^{18} \mathrm{~F}\right]$ fluorodeoxyglucose positron emission tomography and computed tomography. Hum Gene Ther. 2012; 23(1):91-97.

59. Xu F, Li S, Li XL, et al. Phase I and biodistribution study of recombinant adenovirus vector-mediated herpes simplex virus thymidine kinase gene and ganciclovir administration in patients with head and neck cancer and other malignant tumors. Cancer Gene Ther. 2009;16(9):723-730.

60. Li Y, Li LJ, Wang LJ, et al. Selective intra-arterial infusion of rAd-p53 with chemotherapy for advanced oral cancer: a randomized clinical trial. BMC Med. 2014;12:16.

\section{Disclosures}

The authors report no conflict of interest concerning the materials or methods used in this study or the findings specified in this paper.

\section{Author Contributions}

Conception and design: all authors. Acquisition of data: Kan, Srinivasan. Analysis and interpretation of data: all authors. Drafting the article: Kan, Srinivasan. Critically revising the article: all authors. Reviewed submitted version of manuscript: Kan, Srinivasan. Study supervision: Kan, Lang.

\section{Correspondence}

Peter Kan: University of Texas Medical Branch, Galveston, TX. ptkan@utmb.edu. 